\title{
Staining of Sputum for Pneumocystis
}

National Cancer Institute

\section{Source}

National Cancer Institute. Staining of Sputum for Pneumocystis. NCI Thesaurus. Code C23039.

A simple and noninvasive procedure for Pneumocystis jirovecii pneumonia (PCP) diagnosis, in which the specimen used is induced sputum. The specimen is stained with either Giemsa, silver stain or toluidine blue. Alternatively, an indirect immunofluorescent stain using monoclonal antibodies can be used. 